ARTICLE

\title{
Stability and molecular pathways to the formation of spin defects in silicon carbide
}

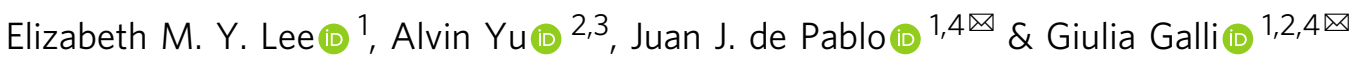

Spin defects in wide-bandgap semiconductors provide a promising platform to create qubits for quantum technologies. Their synthesis, however, presents considerable challenges, and the mechanisms responsible for their generation or annihilation are poorly understood. Here, we elucidate spin defect formation processes in a binary crystal for a key qubit candidatethe divacancy complex ( $\mathrm{VV}$ ) in silicon carbide ( $\mathrm{SiC}$ ). Using atomistic models, enhanced sampling simulations, and density functional theory calculations, we find that $\mathrm{V} V$ formation is a thermally activated process that competes with the conversion of silicon $\left(V_{S_{i}}\right)$ to carbon monovacancies $\left(V_{C}\right)$, and that $V V$ reorientation can occur without dissociation. We also find that increasing the concentration of $V_{S i}$ relative to $V_{C}$ favors the formation of divacancies. Moreover, we identify pathways to create spin defects consisting of antisite-double vacancy complexes and determine their electronic properties. The detailed view of the mechanisms that underpin the formation and dynamics of spin defects presented here may facilitate the realization of qubits in an industrially relevant material.

\footnotetext{
${ }^{1}$ Pritzker School of Molecular Engineering, The University of Chicago, Chicago, IL 60637, USA. ${ }^{2}$ Department of Chemistry, The University of Chicago, Chicago, IL 60637, USA. ${ }^{3}$ Institute for Biophysical Dynamics and James Franck Institute, The University of Chicago, Chicago, IL 60637, USA. ${ }^{4}$ Argonne

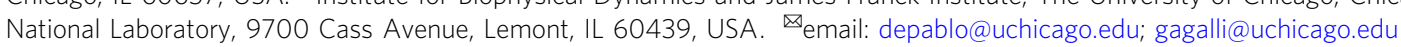


S olid-state spin defects are an emerging platform with applications in quantum information science, sensing, and metrology ${ }^{1}$. The negatively charged nitrogen-vacancy centers $\left(\mathrm{NV}^{-}\right)$in diamond and neutral divacancy defects $(\mathrm{VV})$ in silicon carbide $(\mathrm{SiC})$ represent promising examples of defect complexes for use as spin qubits ${ }^{2-4}$. Divacancies in $\mathrm{SiC}-$ a widely used material in the semiconductor industry-are particularly attractive, as they offer all-optical spin initialization and readout capabilities 5,6 , nuclear spin $\operatorname{control}^{7}$, and a near-infrared highfidelity spin-photon interface ${ }^{8}$, in addition to long coherence times 9

The optical and electronic properties of spin defects in $\mathrm{SiC}$, including the silicon vacancy $\left(\mathrm{V}_{\mathrm{Si}}\right)^{10,11}$, NV centers ${ }^{12,13}$, and carbon antisite-vacancy complexes $\left(\mathrm{C}_{\mathrm{Si}} \mathrm{V}_{\mathrm{C}}\right)^{14}$, have been characterized using a variety of techniques. These include densityfunctional theory (DFT) calculations, electron paramagnetic resonance spectroscopy (EPR), deep-level transient spectroscopy (DLTS), and photoluminescence spectroscopy (PL) ${ }^{15-21}$. Little is known, however, of how to control the selective formation and spatial localization of defect complexes. Addressing this challenge is critical for their integration with optical and electric devices and nanostructures ${ }^{18}$.

Divacancies and other spin defects in SiC are generally generated by ion implantation or electron irradiation, followed by thermal annealing $22-26$. The vacancy-to-VV conversion efficiency, however, is low-a few percent or less 22,27 . VV localization is difficult to control, as samples need to be thermally annealed at high temperatures (above $\sim 700^{\circ} \mathrm{C}$ ) to generate the defect mobility necessary to create divacancies ${ }^{8}$. Furthermore, the spatial placement of $\mathrm{VV}$ appears to depend on the initial distribution of several intrinsic and extrinsic defect species (e.g., vacancies, interstitials, substitutional defects, and dopants) and the Fermi level of the system ${ }^{27}$.

The dynamics of vacancy-defect complexes in semiconductors, particularly $\mathrm{SiC}$, present considerable challenges for theory and computation as well. In particular, the activation energies for vacancy migration in $\mathrm{SiC}$ can be on the order of several electronvolts ${ }^{19}$, thereby limiting the applicability of firstprinciples molecular dynamics (FPMD) simulations. As a result, prior DFT calculations in $\mathrm{SiC}$ have focused mainly on computing defect-formation energies and migration barriers for monovacancies at $T=0 \mathrm{~K}^{28-33}$. Recent kinetic Monte Carlo simulations considered the dynamics of vacancies in $\mathrm{SiC}^{34}$, but they did so by invoking a priori mechanisms for defect mobilization.

Here, we study how spin defects hosted in vacancy-defect complexes are formed in cubic, 3C-SiC, by coupling FPMD simulations with a neural-network-based enhanced sampling technique ${ }^{35,36}$ to efficiently probe phase space, and we compute the free energies and stabilities of several defects. In some cases, our FPMD results are augmented by classical MD simulations conducted with larger system sizes and over longer timescales. Additionally, we predict previously unidentified spin defects consisting of antisites and double vacancies. We focused on the $3 \mathrm{C}$ polytype, given the increasing number of low-cost synthesis strategies developed for high-purity 3C-SiC, e.g., heteroepitaxial growth on silicon substrates 37,38 . Furthermore, $3 \mathrm{C}$-SiC contains a single configuration for each vacancy defect $\left(\mathrm{V}_{\mathrm{Si}}, \mathrm{V}_{\mathrm{C}}\right.$, and $\left.\mathrm{VV}\right)$, and it is thus simpler than other polytypes (e.g., $4 \mathrm{H}-\mathrm{SiC})$ that can host multiple configurations. We find that the formation of divacancies is initiated by the migration and association of monovacancies, which occur in the same temperature regime as the crystallographic reorientation of VV. Our results reveal that a divacancy is a thermodynamically stable state, while $\mathrm{V}_{\mathrm{Si}}$ represents a kinetically trapped state that readily transforms into an intermediate carbon antisite-vacancy $\left(\mathrm{C}_{\mathrm{Si}} \mathrm{V}_{\mathrm{C}}\right)$ defect. Our simulations also show that divacancy formation can be maximized by choosing initial conditions corresponding to a large fraction of $\mathrm{V}_{\mathrm{Si}}$ in the sample to mobilize monovacancies prior to the destabilization of $\mathrm{V}_{\mathrm{Si}}$.

\section{Results}

Divacancy dynamics at high temperatures. The simulation of defect migration in covalently bonded materials, where activation energies may be as large as several electron volts, requires the collection of statistics for $\sim 10-100$ ns. Therefore, capturing the migration of vacancies in $\mathrm{SiC}$ using straightforward FPMD is at present prohibitively demanding from a computational standpoint. Hence, before conducting FPMD simulations coupled to enhanced sampling techniques, we explored the dynamics of vacancy defects in $\mathrm{SiC}$ using an empirical force field ${ }^{39}$, based on a widely used Tersoff-type bond-order formalism ${ }^{40,41}$. The force field was selected for its ability to yield temperature-dependent bulk densities and a decomposition temperature in agreement with experiments ${ }^{42,43}$ (see Supplementary Fig. 1). We found that classical MD using the empirical force field and FPMD simulations yields qualitatively similar energetics for vacancy migration processes (see Supplementary Note 1 for a detailed comparison between the two methods).

To probe the dynamics of $\mathrm{VV}$ and its formation from singlevacancy defects (see Fig. 1), which are only present in low concentrations, we carried out 10 -ns-long classical MD simulations of a 4096-atom supercell of 3C-SiC containing a pair of $\mathrm{V}_{\mathrm{Si}}$ and $\mathrm{V}_{\mathrm{C}}$ (see "Methods"). Analyses of the MD trajectories reveal one or more of the following phenomena at temperatures between $1000 \mathrm{~K}$ and $1500 \mathrm{~K}$ : (1) monovacancy migration (Supplementary Movie 1), (2) VV formation from the pairing of two monovacancies (Fig. 2a-d, Supplementary Movie 3), (3) VV orientational changes (Fig. 2e-h, Supplementary Movie 4), and (4) conversion of $\mathrm{V}_{\mathrm{Si}}$ into a carbon vacancy-antisite complex, $\mathrm{C}_{\mathrm{Si}} \mathrm{V}_{\mathrm{C}}$ (Fig. 2i, j, Supplementary Movie 2). Below $1000 \mathrm{~K}$, we do not observe any vacancy diffusion, even after continuing our simulations for $100 \mathrm{~ns}$; above $1500 \mathrm{~K}$, divacancies dissociate into $\mathrm{V}_{\mathrm{Si}}$ and $\mathrm{V}_{\mathrm{C}}$, and $\mathrm{C}_{\mathrm{Si}} \mathrm{V}_{\mathrm{C}}$ also dissociates into $\mathrm{C}_{\mathrm{Si}}$ and $\mathrm{V}_{\mathrm{C}}$ (Fig. $2 \mathrm{j}-\mathrm{l}$ ). These results suggest that the formation of a $\mathrm{VV}$ is a temperatureactivated process. VV generation occurs in the same temperature regime as the single-vacancy diffusion and, surprisingly, by the destabilization of silicon vacancies via the conversion of $\mathrm{V}_{\mathrm{Si}}$ into $\mathrm{C}_{\mathrm{Si}} \mathrm{V}_{\mathrm{C}}$.

Our results between $\sim 1000 \mathrm{~K}$ and $1500 \mathrm{~K}$ are consistent with recent measurements performed on irradiated samples of $4 \mathrm{H}$ $\mathrm{SiC}^{44}$. These annealing experiments reported that with increasing

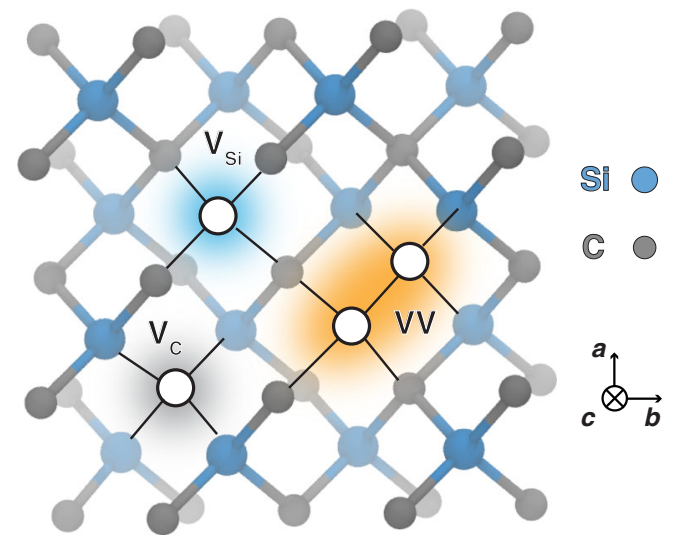

Fig. 1 Ball-and-stick representation of defects in cubic SiC (3C-SiC). A divacancy-defect complex $(\mathrm{VV})$ consists of a carbon vacancy $\left(\mathrm{V}_{\mathrm{C}}\right)$ paired with a silicon vacancy $\left(\mathrm{V}_{\mathrm{Si}}\right)$. Carbon and silicon atoms are shown as gray and blue spheres, respectively, whereas vacancies are depicted as white solid circles. 
Divacancy formation

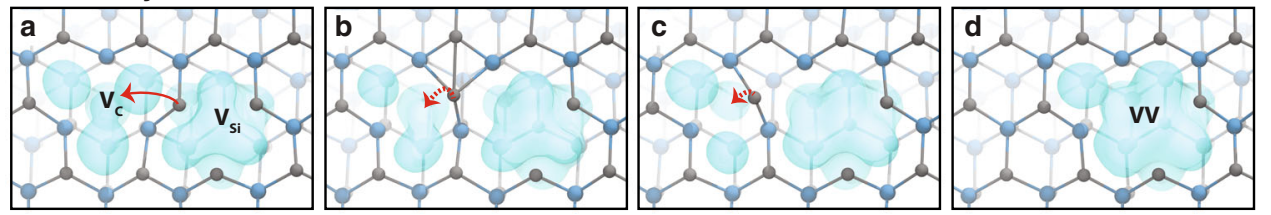

Divacancy reorientation
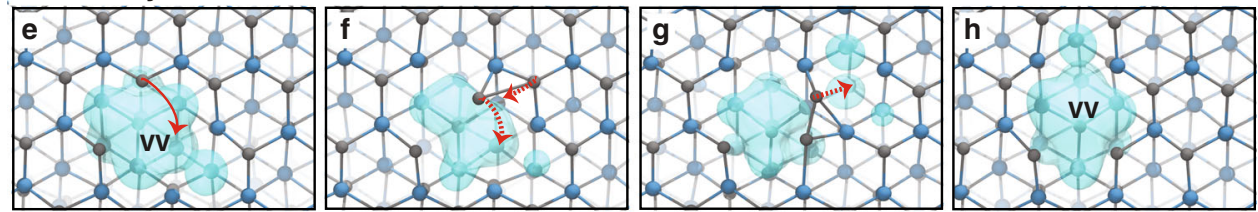

$\mathrm{v}_{\mathrm{si}}$ to $\mathrm{v}_{\mathrm{c}}$ conversion
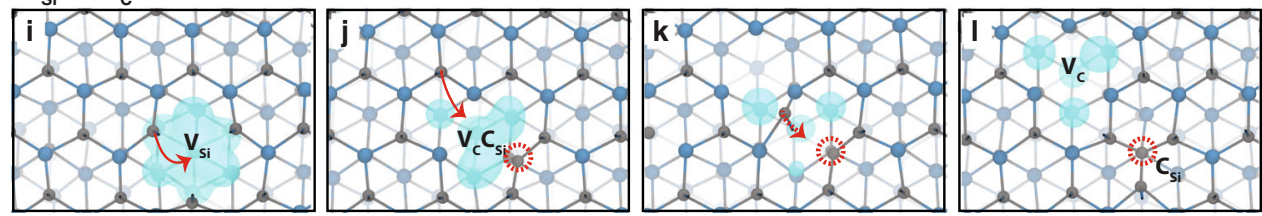

Fig. 2 Dynamics of vacancy formation and migration from classical molecular dynamics (MD) simulations. A series of snapshots from classical MD simulations at temperatures above $1000 \mathrm{~K}$ show the mechanisms for $\mathbf{a}-\mathbf{d}$, the divacancy formation, $\mathbf{e}-\mathbf{h}$, divacancy reorientation, and $\mathbf{i}-\mathbf{I}$, conversion from a $V_{\mathrm{Si}}$ to a $V_{\mathrm{C}}$ before the $\mathrm{V}_{\mathrm{Si}}$ encounters an existing $\mathrm{V}_{\mathrm{C}}$. Three-dimensional vacancy volumes colored in teal blue show the location, size, and shape of void volumes at the defect site. $\mathbf{a} A$ carbon atom in-between the $V_{C}$ and $V_{S i}$ sites displaces the $V_{C}$ and forms a $V V$, following the path marked by a red arrow. b, c During intermediate steps, the mobile carbon atom interacts with neighboring atoms as the local coordination changes from five- and threefold. $\mathbf{d}$ The divacancy consists of a carbon vacancy adjacent to a silicon vacancy. $\mathbf{e}$ The orientation of $V V$ changes as a carbon atom adjacent to $V_{S i}$ migrates to the $V_{C}$ site. $\mathbf{f}, \mathbf{g} \mathrm{C}-\mathrm{C}$ bonds are formed and broken during intermediate steps. $\mathbf{h}$ The $\mathrm{V}_{\mathrm{Si}}-\mathrm{V}_{\mathrm{C}}$ axis in the final $\mathrm{VV}$ configuration is rotated compared to that in the initial configuration, within the laboratory frame. $\mathbf{i}, \mathbf{j} V_{\mathrm{Si}}$ converts into $\mathrm{C}_{\mathrm{Si}} \mathrm{V}_{\mathrm{C}}$ as a nearest-neighbor carbon atom displaces it. Red circle indicates the $\mathrm{C}_{\mathrm{Si}}$ site. k, I $C_{S i} V_{C}$ dissociates into $V_{C}$ and $C_{S i}$ as $V_{C}$ exchanges position with a nearest carbon atom.

temperature above $\sim 600 \mathrm{~K}$, there is a strong correlation between decreasing deep-level transient-spectroscopy (DLTS) intensities of $\mathrm{V}_{\mathrm{Si}}$ and $\mathrm{C}_{\mathrm{Si}} \mathrm{V}_{\mathrm{C}}$ and increasing DLTS and PL intensities of $\mathrm{V}_{\mathrm{C}}$ and VV. The experiments, therefore, are also consistent with the formation of $\mathrm{VV}$ and the formation of $\mathrm{V}_{\mathrm{C}}$ from $\mathrm{V}_{\mathrm{Si}}$ and $\mathrm{C}_{\mathrm{Si}} \mathrm{V}_{\mathrm{C}}$.

Mechanism of VV formation and reorientation processes. Snapshots from our MD simulations above $1000 \mathrm{~K}$ are shown in Fig. 2. We provide a $3 \mathrm{D}$ representation of the free volume at defect sites (see Methods) to investigate changes to the local structure proximal to each defect. As shown in Fig. 2a, b, the size and shape of the free volume surrounding $V_{C}$ and $V_{S i}$ are different. In particular, $V_{C}$ sites have tetrahedrally shaped voids, while $\mathrm{V}_{\mathrm{Si}}$ sites have void volumes consisting of two overlapping tetrahedra centered at the $\mathrm{V}_{\mathrm{Si}}$ site. The void volume of the $\mathrm{V}_{\mathrm{Si}}$ site is larger than that of the $\mathrm{V}_{\mathrm{C}}$ site, indicating that silicon and carbon atoms surrounding $\mathrm{V}_{\mathrm{Si}}$ are more likely to be mobile than the atoms surrounding $\mathrm{V}_{\mathrm{C}}$. When an atom in-between $\mathrm{V}_{\mathrm{C}}$ and $\mathrm{V}_{\mathrm{Si}}$ fills in a vacancy site, the two monovacancies join together to form a VV.

Our MD simulations show that the thermal annealing of $\mathrm{SiC}$ samples may also lead to a reorientation of the $\mathrm{VV}$ without dissociating the original $\mathrm{VV}$, i.e., it leads to a change in the direction of the $\mathrm{V}_{\mathrm{Si}}-\mathrm{V}_{\mathrm{C}}$ axis within the laboratory frame. A recent photoluminescence confocal microscopy study of $\mathrm{NV}^{-}$in diamond reported a crystallographic reorientation process, where the NV-defect symmetry axis in a thermally annealed sample was rotated from its starting configuration (before annealing) in the original reference frame ${ }^{45}$. Our results suggest that thermal annealing at temperatures between $\sim 1000 \mathrm{~K}$ and $1500 \mathrm{~K}$ may be a route toward aligning the orientation of VVs, which would be desirable for sensing applications ${ }^{46,47}$.
Free-energy landscape for divacancy formation and interconversions. To quantify the energetics of defect-interconversion processes and identify possible transition pathways, we computed the free energy or the potential of mean force (PMF) for VV formation at $1500 \mathrm{~K}$ and compared the PMF with free-energy profiles of other defect transformation processes that occur at the same temperature. In order to do so, we turned to DFT calculations, and we computed the PMFs by combining a neural-network-based enhanced sampling method with FPMD ${ }^{36}$, and we used the Cartesian coordinates of the mobile carbon or silicon atom as order parameters. We performed simulations at a Fermi level known to favor the formation of neutral divacancies (see Methods). As expected, the computed 2D-PMFs for defect transformations in 3C$\mathrm{SiC}$ reveal multiple minima and maxima that depend on the specific location of the defects in the crystal structure (Fig. 3a-c).

In the free-energy profile shown in Fig. 3a and Supplementary Fig. 6a, we identify a transition state $(\mathrm{T})$ separating the freeenergy basins of $\mathrm{VV}$ and that of the two unassociated vacancies $\left(\mathrm{V}_{\mathrm{Si}}+\mathrm{V}_{\mathrm{C}}\right)$. We find that the free energy of the $\mathrm{VV}$ is $\sim 1.5 \mathrm{eV}$ lower than that of the $\left(\mathrm{V}_{\mathrm{Si}}+\mathrm{V}_{\mathrm{C}}\right)$, thus confirming that divacancies are stable species at $1500 \mathrm{~K}$, consistent with hightemperature annealing experiments of as-grown $4 \mathrm{H}-\mathrm{SiC}$ samples ${ }^{25,48}$. Additionally, the free-energy difference between the VV and the transition state is comparable to that of the monovacancy migration barrier $\left(\sim 3.0 \mathrm{eV}\right.$ for the $\mathrm{V}_{\mathrm{Si}}$ diffusion and $\sim 3.9 \mathrm{eV}$ for $\mathrm{V}_{\mathrm{C}}$ diffusion, see Supplementary Fig. 5) as both processes involve a next-nearest-neighbor atom substituting a vacancy site. The predicted $\mathrm{V}_{\mathrm{C}}$ migration barrier obtained from FPMD $(\sim 3.9 \mathrm{eV})$ agrees with the barrier measured by DTLS on annealed $\mathrm{SiC}$ samples $(\sim 3.7-4.2 \mathrm{eV})^{19}$.

Regarding the VV reorientation process, our simulations indicate that the diffusion free-energy barriers of $\mathrm{C}$ atoms atoms (Fig. 3d, e) are lower than that of the Si atom (Supplementary 

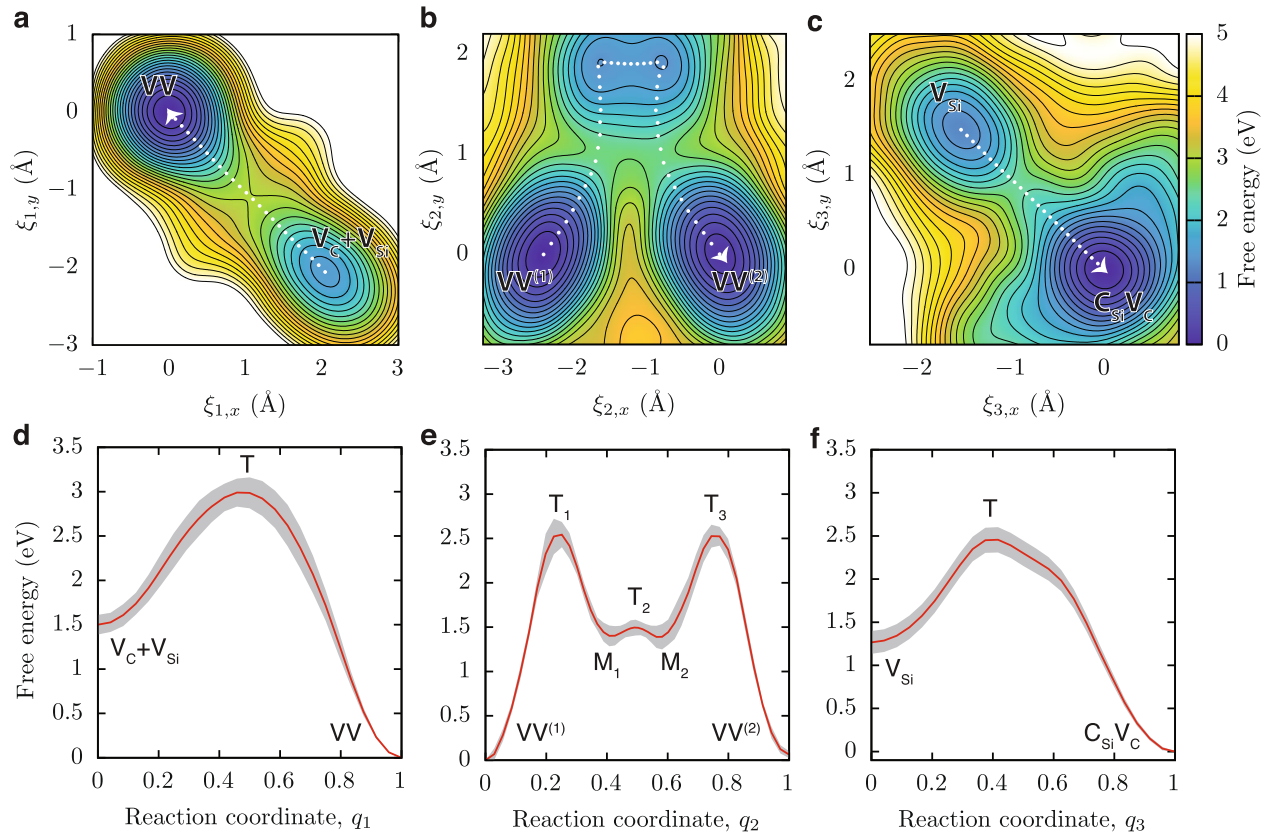

Fig. 3 Free-energy landscapes of vacancy conversion processes for $\mathbf{V V}$ and $\mathbf{V}_{\mathbf{s i}}$. Potentials of mean force (PMFs) were computed using enhanced sampling simulations with FPMD. Two-dimensional order parameters, $\xi_{i}=\left(\xi_{i, x}, \xi_{i, y}\right)$, are used to describe the position of a carbon atom migrating toward a vacancy site, which is the primary mechanism for $\mathrm{VV}$ formation $(\mathbf{a}, \mathbf{d}), \mathrm{VV}$ reorientation $(\mathbf{b}, \mathbf{e})$, and $\mathrm{V}_{\mathrm{Si}}$ to $\mathrm{C}_{\mathrm{Si}} \mathrm{V}_{\mathrm{C}}$ conversion processes $(\mathbf{c}, \mathbf{f})$. a-c $2 \mathrm{D}-\mathrm{PMFs}$ show free energy surfaces of vacancy-conversion processes at $1500 \mathrm{~K}$, whose minimum free-energy pathways are marked by white dotted lines. $\mathbf{d}$-f $\mathbf{f}$ Freeenergy profiles reveal intermediate (state $M_{i}$ ) and transition states (state $T_{i}$ ) along the reaction coordinates. The gray shaded regions denote the error in the PMF determined by block averaging. All three mechanisms are thermally activated processes with relatively high-energy barriers ( $>1.3 \mathrm{eV}$ ).

Fig. 6b) by $\sim 0.4 \mathrm{eV}$. The PMF for the former process features two energetically equivalent local minima $\left(\mathrm{M}_{1}\right.$ and $\left.\mathrm{M}_{2}\right)$ along the freeenergy profile (Fig. 3d). By comparing the free-energy profiles for $\mathrm{VV}$ formation and reorientation processes (Fig. 3d, e), we find that the barrier for $\mathrm{VV}$ dissociation $\left(\mathrm{VV} \rightarrow \mathrm{V}_{\mathrm{Si}}+\mathrm{V}_{\mathrm{C}}\right.$ ) is $\sim 0.5 \mathrm{eV}$ higher than that required to change its orientation. This result suggests that the reorientation of divacancies can occur at a temperature lower than the temperature at which divacancies dissociate.

First-principles free-energy calculations reveal that the height of the barrier for $\mathrm{V}_{\mathrm{Si}}$ conversion into $\mathrm{C}_{\mathrm{Si}} \mathrm{V}_{\mathrm{C}}(\sim 1.3 \mathrm{eV})$ is similar to that of the barrier for $\mathrm{VV}$ formation $(\sim 1.5 \mathrm{eV})$ (see Fig. 3d, f). Moreover, the activation energy for the formation of $\mathrm{C}_{\mathrm{Si}} \mathrm{V}_{\mathrm{C}}$ from $\mathrm{V}_{\mathrm{Si}}$ is lower than that of the migration of $\mathrm{V}_{\mathrm{Si}}(\sim 3.0 \mathrm{eV}$ barrier; see Supplementary Fig. $5 b$ ). Thus, $\mathrm{C}_{\mathrm{Si}} \mathrm{V}_{\mathrm{C}}$ formation is more likely to occur than silicon migration. Interestingly, the free energy of $\mathrm{C}_{\mathrm{Si}} \mathrm{V}_{\mathrm{C}}$ is lower than that of $\mathrm{V}_{\mathrm{Si}}$ by $\sim 1.3 \mathrm{eV}$ at $1500 \mathrm{~K}$.

Optimal temperatures for divacancy stability in as-grown and irradiated SiC. To understand the behavior of divacancies at elevated temperatures, we first analyze the effect of annealing temperatures on the population of VVs in our classical MD simulations. As shown in Fig. 4a, the VV population decreases with increasing temperature above $1700 \mathrm{~K}$, which is consistent with annealing experiments that measured the population of VV of as-grown $\mathrm{SiC}$ samples using electron paramagnetic resonance spectroscopy $(\mathrm{EPR})^{48}$. We find that the kinetics of $\mathrm{VV}$ decay is faster in the presence of a higher-vacancy concentration in the sample (see Supplementary Fig. 8). Hence, it is likely that the VV decay observed experimentally is slower than the one found here, since the VV concentrations of the experimental samples are much lower than those considered in our simulations (see Methods). By fitting an Arrhenius equation to the experimental data, i.e., $y \propto \exp \left(-\triangle E / k_{B} T\right)$ (see Fig. 4a), we estimate the energy difference between a bound and a dissociated VV state to be $\sim 2.1 \mathrm{eV}$. This value is on a similar order of magnitude as the free-energy difference between $\mathrm{VV}$ and $\mathrm{V}_{\mathrm{Si}}+\mathrm{V}_{\mathrm{C}}$ obtained from FPMD $(\sim 1.5 \mathrm{eV})$.

After collecting classical MD trajectories for $10 \mathrm{~ns}$ starting with stable VVs, we find that multiple vacancy defects are formed as divacancies dissociate (Fig. 4b), leading to the presence of $V_{C}, V_{S i}$, and $\mathrm{C}_{\mathrm{Si}} \mathrm{V}_{\mathrm{C}}$. The dominant species detected at high temperatures are carbon vacancies. Other defects $\left(\mathrm{V}_{\mathrm{Si}}\right.$ and $\left.\mathrm{C}_{\mathrm{Si}} \mathrm{V}_{\mathrm{C}}\right)$ are present in smaller fractions (less than $\sim 10 \%$ after $10 \mathrm{~ns}$ ), as they can convert to $\mathrm{V}_{\mathrm{C}}$ via the following process: $\mathrm{V}_{\mathrm{Si}} \rightarrow \mathrm{C}_{\mathrm{Si}} \mathrm{V}_{\mathrm{C}} \rightarrow \mathrm{C}_{\mathrm{Si}}+\mathrm{V}_{\mathrm{C}}$. In order to validate our classical $\mathrm{MD}$ results, we calculated energy differences using DFT, and then we computed defect populations via kinetic Monte Carlo (KMC) simulations (see Supplementary Note 2). Similar to classical MD simulations, our KMC model shows that, at high temperatures, carbon vacancies are the dominant species (Fig. 4b). However, the KMC model predicts that the temperature required to dissociate $\mathrm{C}_{\mathrm{Si}} \mathrm{V}_{\mathrm{C}}$ is higher than that found in classical $\mathrm{MD}$, because the $\mathrm{V}_{\mathrm{C}}$ migration barrier is higher in FPMD $(\sim 3.9 \mathrm{eV})$ than in classical MD $(\sim 2.5 \mathrm{eV})$. The results from $\mathrm{MD}$ and KMC simulations of double vacancies and the computed free-energy landscape of VV formation demonstrate that temperature facilitates both the creation and dissociation of $\mathrm{VV}$. We expect the optimal temperature regime to anneal defects and generate VV to be between $\sim 1000 \mathrm{~K}$ and $\sim 1500 \mathrm{~K}$ or $1250 \pm 250 \mathrm{~K}$. This finding is consistent with recent annealing experiments carried out for irradiated or ion-implanted samples of $4 \mathrm{H}-\mathrm{SiC}^{6,44}$, in which $\mathrm{PL}$ data of $\mathrm{VV}$ also suggest that the concentration of VV defects is maximized at $\sim 1200 \mathrm{~K}$ (Fig. $4 \mathrm{c}$ ). More specifically, by fitting a Gaussian process model to experimental data, we estimate the optimal annealing temperature in experimentally irradiated samples to be $\sim 1193 \mathrm{~K}$.

Compositional dependence in divacancy formation. Our simulations and free-energy calculations show that the kinetics of the $\mathrm{VV}$ formation is controlled by the diffusion of 
a

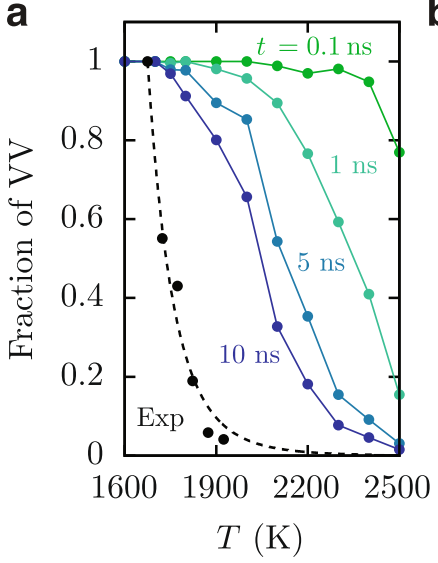

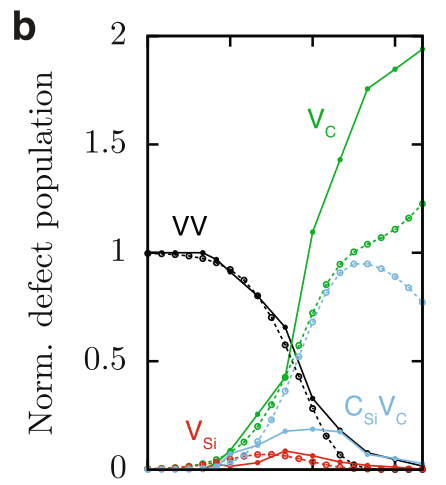

1500180021002400

$T(\mathrm{~K})$

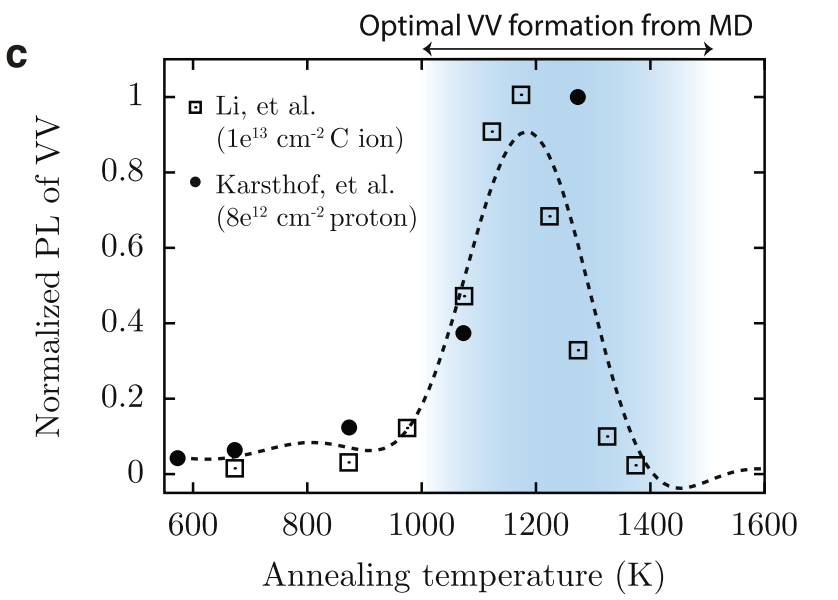

Fig. 4 Effects of temperature on the divacancy formation and

destabilization. a Fraction of divacancies computed from classical MD simulations up to $10 \mathrm{~ns}$, starting with only VVs. The black circles are experimental data, showing normalized EPR intensity of $\mathrm{V} V$ measured after annealing a nonirradiated $4 \mathrm{H}-\mathrm{SiC}$ sample for $30 \mathrm{~min}^{48}$. The dotted black line is a fit to experimental data (see descriptions in the text). Both the simulations and the experiment indicate that divacancies begin to dissociate at $\sim 1700$ K. b Population of defects after VV dissociation. Solid and dotted lines indicate classical MD (10-ns long) and kinetic Monte Carlo (KMC) $\left(10^{15} k_{0}{ }^{-1}\right.$-long) simulations, respectively. Both sets of data demonstrate that $\mathrm{V}_{C}$ 's are the dominant defect species at high temperatures. At each temperature, the number of defects created from $\mathrm{VV}$-dissociation process $\left(\mathrm{V}_{\mathrm{C}}, \mathrm{V}_{\mathrm{Si}}\right.$, and $\left.\mathrm{C}_{\mathrm{Si}} \mathrm{V}_{\mathrm{C}}\right)$ is normalized by the initial number of $\mathrm{VVs}$. $\mathbf{c}$ Photoluminescence $(\mathrm{PL})$ intensity of $\mathrm{VV}$ versus annealing temperature based on data from recent experimental studies using irradiated or ion-implanted samples of $4 \mathrm{H}-\mathrm{SiC}^{6,44}$. The $\mathrm{PL}$ intensity in each data set is normalized by the maximum signal measured. The type of implantation ion and the dose are shown in the legend. The dotted line is the fitted curve using a Gaussian process model, which predicts an optimum annealing temperature of $\sim 1193 \mathrm{~K}$. The temperature regime for optimal VV formation from MD simulations is between $\sim 1000 \mathrm{~K}$ and $\sim 1500 \mathrm{~K}$ (blue shaded region).

monovacancies, while the maximum number of divacancies is limited by the stability of $\mathrm{V}_{\mathrm{Si}}$. These results suggest that the annealing temperature and relative concentration of $\mathrm{V}_{\mathrm{Si}}$ to $\mathrm{V}_{\mathrm{C}}$ affect the mono-to-divacancy conversion efficiency. Experimentally, the composition of point defects in the sample can be changed by growing either carbon-rich or silicon-rich samples ${ }^{49}$ or by controlling the Fermi level ${ }^{21}$. Thus, it is of interest to compute the mono-to-divacancy conversion efficiency at varying ratios of $\mathrm{V}_{\mathrm{Si}}$ to $\mathrm{V}_{\mathrm{C}}$ (see Methods and Supplementary Note 2).

In the KMC simulations carried out in the temperature range where the conversion $\mathrm{C}_{\mathrm{Si}} \mathrm{V}_{\mathrm{C}} \rightarrow \mathrm{C}_{\mathrm{Si}}+\mathrm{V}_{\mathrm{C}}$ occurs, we find that higher concentrations of $\mathrm{V}_{\mathrm{Si}}$ lead to an increase of the probability of $\mathrm{VV}$ formation, even in the absence of $\mathrm{V}_{\mathrm{C}}$ at $t=0$. These results are in agreement with those of classical MD simulations at $1500 \mathrm{~K}$ (see Fig. 5a) and are intriguing, as one would expect no stable VV when the system has predominantly one type of vacancy $\left(\mathrm{V}_{\mathrm{C}}\right.$ or $\mathrm{V}_{\mathrm{Si}}$ ). The results obtained at lower temperature are more intuitive as the KMC simulations show that the maximum number of divacancies is formed when there is an equal concentration of $\mathrm{V}_{\mathrm{Si}}$ and $\mathrm{V}_{\mathrm{C}}$ in the sample. These findings reveal that at high temperatures, the optimal condition to form VV would be to start with a large initial fraction of $\mathrm{V}_{\mathrm{Si}}$ to mobilize monovacancy migration and $\mathrm{C}_{\mathrm{Si}} \mathrm{V}_{\mathrm{C}}$ dissociation, so as to convert some $\mathrm{V}_{\mathrm{Si}}$ into $\mathrm{V}_{\mathrm{C}}$ and then generate additional $\mathrm{V}_{\mathrm{C}}$, as shown schematically in Fig. 5b. A low initial concentration of $\mathrm{V}_{\mathrm{Si}}$ reduces the $\mathrm{VV}$ conversion efficiency (see Fig. $5 c$ ) because there are no energetically favored pathways to form $V_{S i}$ from $V_{C}$.

MD simulations identify potential spin defects. The generation of $\mathrm{V}_{\mathrm{Si}}$ and $\mathrm{C}_{\mathrm{Si}} \mathrm{V}_{\mathrm{C}}$ species during $\mathrm{MD}$ simulations of $\mathrm{VV}$ dissociation, validated by DFT-based KMC simulations (Fig. 4b), pointed out species that, in appropriate charged states, are interesting spin defects ${ }^{10,14}$. Prior studies have detected and characterized spin defects based on impurities and antisite defects using EPR measurements and DFT calculations at $T=0 \mathrm{~K}^{50,51}$. Remarkably, we also identified potential spin defects from MD simulations of divacancies at $2000 \mathrm{~K}$ and above through the process: $\quad \mathrm{VV} \rightarrow \mathrm{V}_{\mathrm{C}} \mathrm{C}_{\mathrm{Si}} \mathrm{V}_{\mathrm{C}} \rightarrow \mathrm{C}_{\mathrm{Si}} \mathrm{V}_{\mathrm{C}}+\mathrm{V}_{\mathrm{C}}$ (see Supplementary Movie 5). Importantly, simulations show that these defects can be stabilized to room temperature (see Supplementary Note 3). Multiple structures of this vacancy complex are possible, depending on the relative orientation between $\mathrm{C}_{\mathrm{Si}} \mathrm{V}_{\mathrm{C}}$ and $\mathrm{V}_{\mathrm{C}}$, as well as the physical proximity between the two defects.

The results of hybrid DFT calculations ${ }^{52}$ of the electronic structure of the neutral antisite-vacancy complexes shown in Fig. 6, indicate that $\mathrm{V}_{\mathrm{C}} \mathrm{C}_{\mathrm{Si}} \mathrm{V}_{\mathrm{C}}$ (Fig. 6b) and one of the structures for $\left[\mathrm{C}_{\mathrm{Si}} \mathrm{V}_{\mathrm{C}}+\mathrm{V}_{\mathrm{C}}\right],\left[\mathrm{C}_{\mathrm{Si}} \mathrm{V}_{\mathrm{C}}+\mathrm{V}_{\mathrm{C}}\right]_{n=3}$ (Fig. 6c), consist of defect levels with localized electron densities. The lowest electronic configuration of these defect levels is a triplet, as the $\mathrm{NV}^{-}$in diamond and $\mathrm{VV}^{0}$ in $\mathrm{SiC}$. However, we note that in the antisitevacancy complexes, the two carbon vacancies are separated by a carbon antisite and, therefore, their ground-state spin densities are more spatially delocalized than those of the VV state.

In the case of the $\mathrm{V}_{\mathrm{C}} \mathrm{C}_{\mathrm{Si}} \mathrm{V}_{\mathrm{C}}$ state, we find unoccupied defect levels within the bandgap close to the conduction band, similar to neutral $\mathrm{VV}$ spin defect in $3 \mathrm{C}-\mathrm{SiC}^{53}$ (see also Fig. 6a). These results suggest that $\left[\mathrm{C}_{\mathrm{Si}} \mathrm{V}_{\mathrm{C}}+\mathrm{V}_{\mathrm{C}}\right]_{n=3}$ and, especially, $\mathrm{V}_{\mathrm{C}} \mathrm{C}_{\mathrm{Si}} \mathrm{V}_{\mathrm{C}}$ are possible spin defects. Since $\mathrm{V}_{\mathrm{C}} \mathrm{C}_{\mathrm{Si}} \mathrm{V}_{\mathrm{C}}$ and $\left[\mathrm{C}_{\mathrm{Si}} \mathrm{V}_{\mathrm{C}}+\mathrm{V}_{\mathrm{C}}\right]$ are created when $\mathrm{VV}$ dissociates $(T>\sim 1700 \mathrm{~K})$, it is unlikely that these spin defects are simultaneously present with VVs in post-annealed samples. However, if present, high concentrations of these spin defects and/or isolated $\mathrm{C}_{\mathrm{Si}}$ species with localized electronic spins ${ }^{54,55}$ may unfavorably decrease the VV coherence times ${ }^{56}$.

Notably, our simulations did not incorporate any prior information on the formation mechanism of the spin defects found here. This highlights the potential of an atomistic approach to identify not only novel spin defects when combined with electronic-structure calculations but also microscopic mechanisms of their formation.

\section{Discussion}

Our simulations of the relative stability of monovacancies and divacancies in $3 \mathrm{C}-\mathrm{SiC}$ and their dynamics have revealed that, to generate a high concentration of $\mathrm{VV}$ in the material, a large ratio 
a

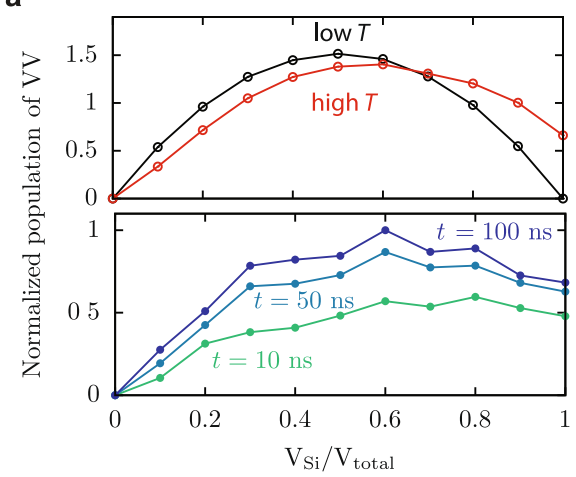

b

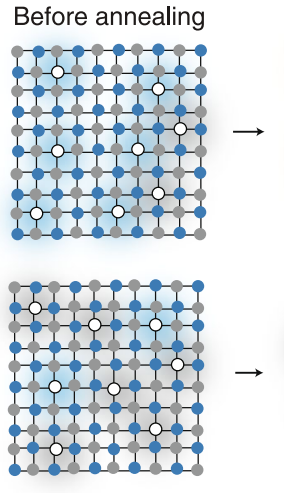

After annealing

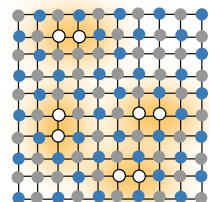

SIO

C 0

$\mathrm{v}_{\text {sI }} \mathrm{O}$

$\mathrm{V}_{\mathrm{c}} \mathrm{O}$

VV $\mathrm{O}$

Fig. 5 Dependence of VV-conversion efficiency on the initial composition of monovacancies. a (top panel) KMC simulations showing changes in the long-time limit of $\mathrm{VV}$ population starting with varying concentrations of $\mathrm{V}_{\mathrm{Si}}$, i.e., the number of $\mathrm{V}_{\mathrm{Si}}$ over the total number of vacancies, at two different temperatures $T$ (black line at $T_{K M C}=1500 \mathrm{~K}$, red line at $T_{K M C}=2000 \mathrm{~K}$ ). (bottom panel) Classical MD simulations showing the time evolution of mono- to divacancy conversion up to $100 \mathrm{~ns}$ at $T_{\mathrm{MD}}=1500 \mathrm{~K}$, starting with different fractions of $\mathrm{V}_{\mathrm{Si}}$, i.e., the number of $\mathrm{V}_{\mathrm{Si}}$ over the total number of $\mathrm{V}_{\mathrm{Si}}$ and $\mathrm{V}_{\mathrm{C}}$. The $\mathrm{VV}$ formation favors higher concentrations of $\mathrm{V}_{\mathrm{Si}}$, agreeing with the high-temperature behavior of the KMC model. b-c Schematics for controlling divacancy formation by tuning the $V_{S i}-t o-V_{C}$ ratio during thermal annealing at high temperature based on the results from panel $\mathbf{a}$. $\mathbf{b}$ Samples having a greater number of $V_{S i}$ than $V_{C}$ prior to annealing (left) producing more $V V$ after annealing (right). c Samples having a fewer number of $V_{S i}$ than $V_{C}$ prior to annealing (left), producing fewer $\mathrm{V} V$ after annealing (right).

a
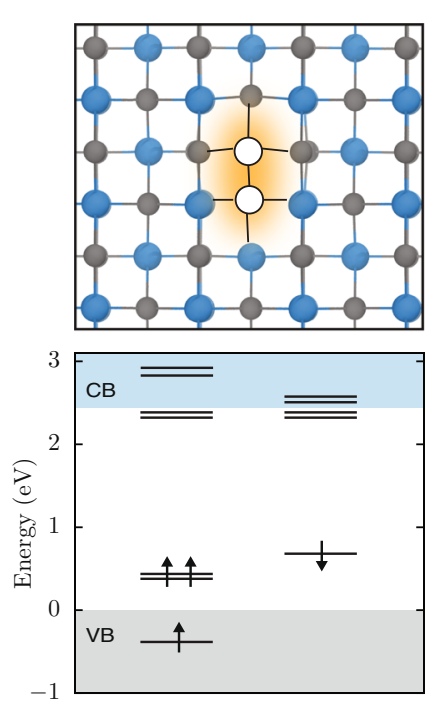

b
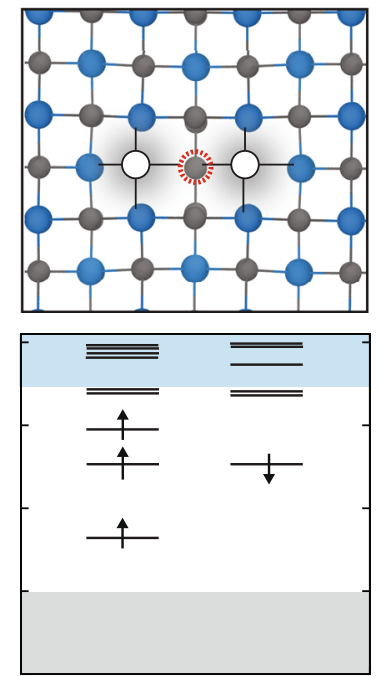

c $\quad\left[\mathrm{C}_{\mathrm{si}} \mathrm{V}_{\mathrm{c}}+\mathrm{V}_{\mathrm{c}}\right]_{\mathrm{n}=3}$
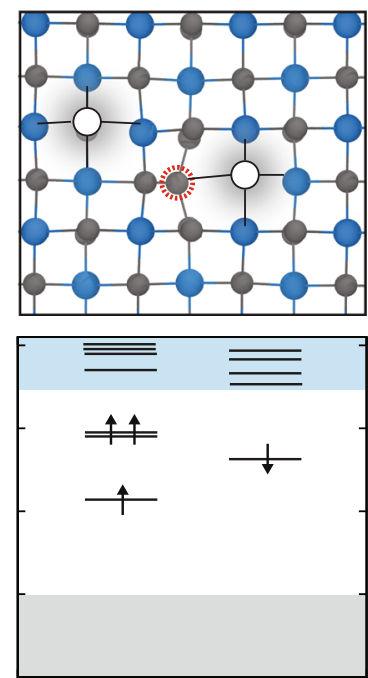

Fig. 6 Electronic structures of $\mathbf{V V}^{\mathbf{0}}$ and antisite-vacancy complexes identified from MD simulations of $\mathbf{V V}$ dissociation. Defect structures (top) and defect energy-level diagrams (bottom) are shown for neutral $\mathbf{a}, \mathrm{VV}, \mathbf{b}, \mathrm{V}_{\mathrm{C}} \mathrm{C}_{\mathrm{Si}} \mathrm{V}_{\mathrm{C}}$, and $\mathbf{c},\left[\mathrm{C}_{\mathrm{Si}} \mathrm{V}_{\mathrm{C}}+\mathrm{V}_{\mathrm{C}}\right]_{n=3}$. For the $\left[\mathrm{C}_{\mathrm{Si}} \mathrm{V}_{\mathrm{C}}+\mathrm{V}_{\mathrm{C}}\right]$ defect shown here, the two carbon vacancies are separated by at least $n=3$ atoms. For each defect, the lowest electronic configuration is a triplet state. Shaded gray areas indicate energy levels below the valence band (VB) and above the conduction band (CB). The spin-majority (spin-minority) channel is denoted by upward(downward-) pointing arrows.

of silicon to carbon-vacancy concentration is needed. Several of the defect-transformation mechanisms observed in our simulations are consistent with and help explain annealing experiments, both in as-grown samples and irradiated/ion-implanted samples.

Divacancies are found to undergo crystallographic reorientation without dissociating. These findings indicate that the optimal annealing temperature for reorientation, whose control is of interest for sensing applications, is lower than that required to generate the defects.

Beyond having delineated the formation mechanisms of $\mathrm{VV}$, $\mathrm{V}_{\mathrm{Si}}$, and $\mathrm{C}_{\mathrm{Si}} \mathrm{V}_{\mathrm{C}}$ spin defects, which arise from the dissociation of $\mathrm{VV}$ into $\mathrm{C}_{\mathrm{Si}} \mathrm{V}_{\mathrm{C}}$ and another $\mathrm{V}_{\mathrm{C}}$, we have also discovered plausible new spin defects and characterized their electronic structure using hybrid density-functional theory. Such defects provide new opportunities for quantum technology applications.

\section{Methods}

Classical MD simulations for divacancy dynamics. Bulk 3C-SiC was modeled as a cubic supercell containing 4096 atoms with 3D periodic boundary conditions. Energy minimization and MD simulations were carried out using LAMMPS ${ }^{57}$ and an environment-dependent interatomic potential (EDIP) force field ${ }^{39}$, chosen after benchmarking several empirical potentials for SiC (Supplementary Fig. 1). We equilibrated a $3 \mathrm{C}-\mathrm{SiC}$ structure at the target temperature $T$ and pressure of $1 \mathrm{~atm}$ in the NPT ensemble for $1 \mathrm{~ns}$ with a 1.0-fs time step. The system was further equilibrated for an additional $1 \mathrm{~ns}$ in the NVT ensemble. A Nose-Hoover thermostat and barostat were used to control temperature and pressure, respectively.

After the 2-ns long equilibration phase of the bulk crystal, three types of NVTproduction runs were conducted with supercells containing one of the following: (1) a pair of $\mathrm{V}_{\mathrm{C}}$ and $\mathrm{V}_{\mathrm{Si}}$ and (2) a divacancy, and (3) 40 monovacancies with varying composition of monovacancies. In the first case, 20 independent 10 -ns-long trajectories were sampled at each $T$ between $500 \mathrm{~K}$ and $2500 \mathrm{~K}$, every $500 \mathrm{~K}$. In the second case, 100 independent 10 -ns-long trajectories starting with a single divacancy were sampled at each temperature between $1500 \mathrm{~K}$ and $2500 \mathrm{~K}$, every $100 \mathrm{~K}$. In the last case, a high-vacancy concentration $(40 / 4096=1 \%)$ was used to increase the 
probability of forming VVs from monovacancies within the simulation time of $100 \mathrm{~ns}$. At each composition (ratio of $\mathrm{V}_{\mathrm{Si}}$ to $\mathrm{V}_{\mathrm{C}}$ ), 25 independent 100-ns-long trajectories were collected at $1500 \mathrm{~K}$. The VV-conversion efficiency at time $t$ was calculated by dividing the number of divacancies at time $t$ by half of the number of monovacancies at $t=0$. Overall, we performed over $2200 \mathrm{MD}$ simulations of $10-100 \mathrm{~ns}$ each.

Void-volume analysis for vacancy defects. To identify and track the position of vacancy defects, we constructed Voronoi cells around each atom in a bulk crystal using VORO $++{ }^{58}$. The location of the vacancy defect was defined by the centroid position of an empty Voronoi cell after aligning the atoms in the bulk crystal to those in the MD snapshot. We computed the 3D void volume at the vacancy-defect site by calculating the distribution of voids on a discretized $3 \mathrm{D}$ grid with a spacing of $0.15 \AA \times 0.15 \AA \times 0.15 \AA$. The excluded volume of each atom was approximated as a sphere with a radius $R(1.6 \AA$ for carbon and $2.0 \AA$ for silicon), where the ratio of the $\mathrm{C}$ and $\mathrm{Si}$ radii was the same as the ratio of their respective van der Waals radii.

\section{Determination of defect-energy levels using DFT calculations. Electronic-}

structure calculations were carried out using DFT as implemented in the Quantum Espresso $^{59}$ and Qbox code ${ }^{60,61}$. Defects were modeled using $4 \times 4 \times 4$ or 512 -atom supercells of $3 \mathrm{C}-\mathrm{SiC}$. The interaction between core and valence electrons was described using Optimized Norm-Conserving Vanderbilt pseudopotentials from the SG15 library ${ }^{62}$ and we used a plane-wave basis with a kinetic energy cutoff of $55 \mathrm{Ry}$; the Brillouin zone was sampled with the $\Gamma$-point. For each defect calculation, a smearing with a width of 0.001 Ry was used in ground-state electronic-structure calculation, using the Marzari-Vanderbilt procedure ${ }^{63}$; geometries were optimized at the generalized gradient approximation (GGA) level of DFT using the Perdew-Burke-Ernzerhof (PBE) functional ${ }^{64}$ in Quantum Espresso. The defectlevel energy diagrams were obtained using the dielectric-dependent hybrid (DDH) functional with a self-consistent Hartree-Fock mixing parameter of $\alpha=0.15$ for $\mathrm{SiC}^{52}$ at the ground-state triplet configuration using the recursive bisection method $^{65}$ as implemented in the Qbox code.

\section{Free-energy calculations by coupling FPMD simulations with enhanced} sampling. The free-energy landscape ${ }^{66-69}$ for defect migration and formation was computed using the combined force-frequency method or CFF-FPMD ${ }^{36}$ as implemented in the software package SSAGES ${ }^{70}$ coupled to the FPMD software Qbox. Unbiased FPMD and enhanced sampling simulations were performed in the NVT ensemble using the Bussi-Donadio-Parrinello thermostat ${ }^{71}$ with a time step of $0.967 \mathrm{fs}$ at $T=1500 \mathrm{~K}$. To evaluate energy and forces, DFT calculations were carried out using the PBE functional using plane waves with a 40-Ry kinetic energy cutoff and the I-point to sample the Brillouin zone. We used the same pseudopotential as in the defect energy-level calculations. For computational efficiency, the system was modeled with a 216-atom cell (see Supplementary Note 4 for the finitesize effect). Defect dynamical properties were simulated for the ground-state triplet configuration. The total charge of the supercell was neutral; however, charge rearrangements (i.e., charges near individual vacancy sites) during vacancy migration are allowed to occur in FPMD simulations. The $x$ - and $y$-coordinates of a mobile atom displacing a vacancy site were used as the collective variables (CV), e.g., $\xi_{i, x}=X_{C_{i}}$ and $\xi_{i, y}=Y_{C_{i}}$ for the $\mathrm{V}_{\mathrm{C}}$ migration. During CFF-FPMD simulations, running average of forces and frequencies was recorded on a $2 \mathrm{D}$ grid with a grid spacing of $0.21 \AA$ along $\xi_{i, x}$ and $\xi_{i, y}$. Bayesian-regularized artificial neural networks (BRANN) with two hidden layers ( 8 and 6 nodes in the first and the second layers, respectively) were used. For each free-energy calculation, up to 63 walkers/replicas were employed, and simulations were carried out up to $30 \mathrm{ps}$ per walker. To compute the mean free-energy path (MFEP), we fitted the 2D-PMF to a BRANN to enable a smooth interpolation of free energies from a discretized 2D-CV grid ${ }^{36}$. The nudge elastic-band method $^{72}$ with a spring constant of $5 \mathrm{eV} / \AA$ was applied to the BRANN-fitted PMF to find the MFEP between the initial and final states.

\section{Data availability}

Data that support the findings of this study will be available through the Qresp ${ }^{73}$ curator at https://paperstack.uchicago.edu/explorer.

Received: 24 March 2021; Accepted: 28 September 2021; Published online: 03 November 2021

\section{References}

1. Awschalom, D. D., Hanson, R., Wrachtrup, J. \& Zhou, B. B. Quantum technologies with optically interfaced solid-state spins. Nat. Photonics 12, 516-527 (2018).

2. Maurer, P. C. et al. Room-temperature quantum bit memory exceeding one second. Science 336, 1283-1286 (2012).

3. Balasubramanian, G. et al. Ultralong spin coherence time in isotopically engineered diamond. Nat. Mater. 8, 383-387 (2009).
4. Anderson, C. P. et al. Electrical and optical control of single spins integrated in scalable semiconductor devices. Science 366, 1225-1230 (2019).

5. Koehl, W. F., Buckley, B. B., Heremans, F. J., Calusine, G. \& Awschalom, D. D. Room temperature coherent control of defect spin qubits in silicon carbide. Nature 479, 84-87 (2011).

6. $\mathrm{Li}$, Q. et al. Room temperature coherent manipulation of single-spin qubits in silicon carbide with a high readout contrast. Natl Sci. Rev. https://doi.org/ 10.1093/nsr/nwab122 (2021)

7. Klimov, P. V., Falk, A. L., Christle, D. J., Dobrovitski, V. V. \& Awschalom, D. D. Quantum entanglement at ambient conditions in a macroscopic solid-state spin ensemble. Sci. Adv. 1, e1501015 (2015).

8. Christle, D. J. et al. Isolated spin qubits in $\mathrm{SiC}$ with a high-fidelity infrared spin-to-photon interface. Phys. Rev. X 7, 021046 (2017).

9. Bourassa, A. et al. Entanglement and control of single nuclear spins in isotopically engineered silicon carbide. Nat. Mater. 19, 1319-1325 (2020).

10. Widmann, M. et al. Coherent control of single spins in silicon carbide at room temperature. Nat. Mater. 14, 164-168 (2015).

11. Niethammer, M. et al. Coherent electrical readout of defect spins in silicon carbide by photo-ionization at ambient conditions. Nat. Commun. 10, 5569 (2019).

12. $\mathrm{Mu}, \mathrm{Z}$. et al. Coherent manipulation with resonant excitation and single emitter creation of nitrogen vacancy centers in $4 \mathrm{H}$ silicon carbide. Nano Lett. 20, 6142-6147 (2020).

13. Wang, J.-F. et al. Coherent control of nitrogen-vacancy center spins in silicon carbide at room temperature. Phys. Rev. Lett. 124, 223601 (2020).

14. Castelletto, S. et al. A silicon carbide room-temperature single-photon source. Nat. Mater. 13, 151-156 (2014).

15. Weber, J. R. et al. Quantum computing with defects. Proc. Natl Acad. Sci. USA 107, 8513-8518 (2010).

16. Ma, H., Govoni, M. \& Galli, G. Quantum simulations of materials on nearterm quantum computers. npj Comput. Mater. 6, 1-8 (2020).

17. Iwamoto, N. \& Svensson, B. G. Chapter Ten - Point Defects in Silicon Carbide. in Semiconductors and Semimetals (eds Romano, L., Privitera, V. \& Jagadish, C.) vol. 91, 369-407 (Elsevier, 2015).

18. Wolfowicz, G. et al. Qubit guidelines for solid-state spin defects. Nat. Rev. Mater. https://doi.org/10.1038/s41578-021-00306-y (2020).

19. Bathen, M. E. et al. Anisotropic and plane-selective migration of the carbon vacancy in SiC: theory and experiment. Phys. Rev. B 100, 014103 (2019).

20. Ayedh, H. M., Bobal, V., Nipoti, R., Hallén, A. \& Svensson, B. G. Formation of carbon vacancy in $4 \mathrm{H}$ silicon carbide during high-temperature processing. $J$. Appl. Phys. 115, 012005 (2014).

21. Son, N. T. \& Ivanov, I. G. Charge state control of the silicon vacancy and divacancy in silicon carbide. J. Appl. Phys. 129, 215702 (2021).

22. Falk, A. L. et al. Polytype control of spin qubits in silicon carbide. Nat. Commun. 4, 1819 (2013).

23. Wang, J. et al. Scalable fabrication of single silicon vacancy defect arrays in silicon carbide using focused ion beam. ACS Photonics 4, 1054-1059 (2017).

24. Wang, J.-F. et al. On-demand generation of single silicon vacancy defects in silicon carbide. ACS Photonics 6, 1736-1743 (2019).

25. Wolfowicz, G. et al. Optical charge state control of spin defects in $4 \mathrm{H}-\mathrm{SiC}$. Nat. Commun. 8, 1876 (2017).

26. Pavunny, S. P. et al. Arrays of $\mathrm{Si}$ vacancies in $4 \mathrm{H}-\mathrm{SiC}$ produced by focused $\mathrm{Li}$ ion beam implantation. Sci. Rep. 11, 3561 (2021).

27. Castelletto, S. \& Boretti, A. Silicon carbide color centers for quantum applications. J. Phys. Photonics 2, 022001 (2020).

28. Bockstedte, M., Mattausch, A. \& Pankratov, O. Ab initio study of the migration of intrinsic defects in 3C-SiC. Phys. Rev. B 68, 205201 (2003).

29. Bockstedte, M., Mattausch, A. \& Pankratov, O. Ab initio study of the annealing of vacancies and interstitials in cubic SiC: vacancy-interstitial recombination and aggregation of carbon interstitials. Phys. Rev. B 69, 235202 (2004).

30. Roma, G., Bruneval, F., Ting, L. A., Bedoya Martínez, O. N. \& Crocombette, J. P. Formation and migration energy of native defects in silicon carbide from first principles: an overview. Defect Diffus. Forum 323-325, 11-18 (2012).

31. Wang, X. et al. Formation and annealing behaviors of qubit centers in $4 \mathrm{H}-\mathrm{SiC}$ from first principles. J. Appl. Phys. 114, 194305 (2013).

32. Rurali, R., Hernández, E., Godignon, P., Rebollo, J. \& Ordejón, P. First principles studies of neutral vacancies diffusion in SiC. Comput. Mater. Sci. 27, 36-42 (2003).

33. Rauls, E., Frauenheim, Th., Gali, A. \& Deák, P. Theoretical study of vacancy diffusion and vacancy-assisted clustering of antisites in SiC. Phys. Rev. B 68 , 155208 (2003).

34. Kuate Defo, R. et al. Energetics and kinetics of vacancy defects in $4 \mathrm{H}-\mathrm{SiC}$ Phys. Rev. B 98, 104103 (2018).

35. Sevgen, E., Guo, A. Z., Sidky, H., Whitmer, J. K. \& de Pablo, J. J. Combined force-frequency sampling for simulation of systems having rugged free energy landscapes. J. Chem. Theory Comput. 16, 1448-1455 (2020). 
36. Lee, E. M. Y. et al. Neural network sampling of the free energy landscape for nitrogen dissociation on ruthenium. J. Phys. Chem. Lett. 12, 2954-2962 (2021).

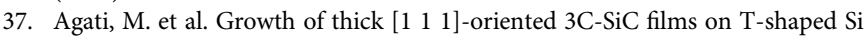
micropillars. Mater. Des. 208, 109833 (2021).

38. Fisicaro, G. et al. Genesis and evolution of extended defects: the role of evolving interface instabilities in cubic SiC. Appl. Phys. Rev. 7, 021402 (2020).

39. Lucas, G., Bertolus, M. \& Pizzagalli, L. An environment-dependent interatomic potential for silicon carbide: calculation of bulk properties, highpressure phases, point and extended defects, and amorphous structures. $J$. Phys. Condens. Matter 22, 035802 (2010).

40. Tersoff, J. New empirical approach for the structure and energy of covalent systems. Phys. Rev. B 37, 6991-7000 (1988).

41. Tersoff, J. Modeling solid-state chemistry: interatomic potentials for multicomponent systems. Phys. Rev. B 39, 5566-5568 (1989).

42. Kern, E. L., Hamill, D. W., Deem, H. W. \& Sheets, H. D. Thermal properties of $\beta$-silicon carbide from 20 to $2000^{\circ} \mathrm{C}$. in Silicon Carbide-1968 S25-S32 (Elsevier, 1969). https://doi.org/10.1016/B978-0-08-006768-1.50007-3.

43. Pierson, H. O. Characteristics and properties of silicon carbide and boron carbide. in Handbook of Refractory Carbides and Nitrides, 137-155 (Elsevier, 1996). https://doi.org/10.1016/B978-081551392-6.50009-X.

44. Karsthof, R., Bathen, M. E., Galeckas, A. \& Vines, L. Conversion pathways of primary defects by annealing in proton-irradiated $\mathrm{n}$-type $4 \mathrm{H}-\mathrm{SiC}$. Phys. Rev. B 102, 184111 (2020).

45. Chakravarthi, S. et al. Window into NV center kinetics via repeated annealing and spatial tracking of thousands of individual NV centers. Phys. Rev. Mater. 4, 023402 (2020)

46. Pham, L. M. et al. Enhanced metrology using preferential orientation of nitrogen-vacancy centers in diamond. Phys. Rev. B 86, 121202 (2012).

47. Hsieh, S. et al. Imaging stress and magnetism at high pressures using a nanoscale quantum sensor. Science 366, 1349-1354 (2019).

48. Carlos, W. E., Garces, N. Y., Glaser, E. R. \& Fanton, M. A. Annealing of multivacancy defects in 4H-SiC. Phys. Rev. B 74, 235201 (2006).

49. Fujiwara, H., Danno, K., Kimoto, T., Tojo, T. \& Matsunami, H. Effects of C/Si ratio in fast epitaxial growth of $4 \mathrm{H}-\mathrm{SiC}(0001)$ by vertical hot-wall chemical vapor deposition. J. Cryst. Growth 281, 370-376 (2005).

50. Carlsson, P. et al. EPR and ab initio calculation study on the EI4 center in $4 \mathrm{H}-$ and 6H-SiC. Phys. Rev. B 82, 235203 (2010).

51. Szász, K. et al. Spin and photophysics of carbon-antisite vacancy defect in $4 \mathrm{H}$ silicon carbide: a potential quantum bit. Phys. Rev. B 91, 121201 (2015).

52. Skone, J. H., Govoni, M. \& Galli, G. Self-consistent hybrid functional for condensed systems. Phys. Rev. B 89, 195112 (2014).

53. Gordon, L., Janotti, A. \& Van de Walle, C. G. Defects as qubits in $3 \mathrm{C}-$ and $4 \mathrm{H}$ - SiC. Phys. Rev. B 92, 045208 (2015).

54. Baranov, P. G., Ilyin, I. V., Soltamova, A. A. \& Mokhov, E. N. Identification of the carbon antisite in SiC: EPR of $13 \mathrm{C}$ enriched crystals. Phys. Rev. B 77, 085120 (2008)

55. Petrenko, T. L. \& Bryksa, V. P. Electronic structure of carbon antisite in SiC: localization versus delocalization. Phys. Rev. B Condens. Matter 517, 47-52 (2017).

56. Bourassa, A. et al. Entanglement and control of single nuclear spins in isotopically engineered silicon carbide. Nat. Mater. 19, 1319-1325 (2020).

57. Plimpton, S. Fast parallel algorithms for short-range molecular dynamics. $J$. Comput. Phys. 117, 1-19 (1995).

58. Rycroft, C. H. VORO++: A three-dimensional Voronoi cell library in $\mathrm{C}++$. Chaos 19, 041111 (2009)

59. Giannozzi, P. et al. QUANTUM ESPRESSO: a modular and open-source software project for quantum simulations of materials. J. Phys. Condens. Matter 21, 395502 (2009).

60. Gygi, F. Architecture of Qbox: a scalable first-principles molecular dynamics code. IBM J. Res. Dev. 52, 137-144 (2008).

61. Qbox. Qbox First-Principles Molecular Dynamics Code. http://qboxcode.org (2020).

62. Schlipf, M. \& Gygi, F. Optimization algorithm for the generation of ONCV pseudopotentials. Comput. Phys. Commun. 196, 36-44 (2015).

63. Marzari, N., Vanderbilt, D., De Vita, A. \& Payne, M. C. Thermal contraction and disordering of the $\mathrm{Al}(110)$ surface. Phys. Rev. Lett. 82, 3296-3299 (1999).

64. Perdew, J. P., Burke, K. \& Ernzerhof, M. Generalized gradient approximation made simple. Phys. Rev. Lett. 77, 3865-3868 (1996).

65. Dawson, W. \& Gygi, F. Performance and accuracy of recursive subspace bisection for hybrid DFT calculations in inhomogeneous systems. J. Chem. Theory Comput. 11, 4655-4663 (2015).

66. Roux, B. The calculation of the potential of mean force using computer simulations. Comput. Phys. Commun. 91, 275-282 (1995).

67. Yu, A. et al. Molecular lock regulates binding of glycine to a primitive NMDA receptor. Proc. Natl Acad. Sci. USA 113, E6786-E6795 (2016).
68. Yu, A., Lee, E. M. Y., Jin, J. \& Voth, G. A. Atomic-scale characterization of mature HIV-1 capsid stabilization by inositol hexakisphosphate (IP6). Sci. $A d v .6$, eabc6465 (2020).

69. Yu, A., Wied, T., Belcher, J. \& Lau, A. Y. Computing conformational free energies of iGluR ligand-binding domains. in Ionotropic Glutamate Receptor Technologies (ed. Popescu, G. K.) 119-132 (Springer New York, 2016). https:// doi.org/10.1007/978-1-4939-2812-5_9

70. Sidky, H. et al. SSAGES: software suite for advanced general ensemble simulations. J. Chem. Phys. 148, 044104 (2018).

71. Bussi, G., Donadio, D. \& Parrinello, M. Canonical sampling through velocity rescaling. J. Chem. Phys. 126, 014101 (2007).

72. Henkelman, G. \& Jónsson, H. Improved tangent estimate in the nudged elastic band method for finding minimum energy paths and saddle points. J. Chem. Phys. 113, 9978-9985 (2000).

73. Govoni, M. et al. Qresp, a tool for curating, discovering and exploring reproducible scientific papers. Sci. Data 6, 190002 (2019).

\section{Acknowledgements}

We thank Chris Anderson, Nazar Delegan, and He Ma for insightful comments and discussions, Joe Heremans for a careful reading of the paper and helpful discussions, and François Gygi for help in setting up FPMD calculations using the Qbox code. We gratefully acknowledge the use of Bebop in the Laboratory Computing Resource Center at Argonne National Laboratory; the computational resources at the University of Chicago Research Computing Center; and the Argonne Leadership Computing Facility, a DOE Office of Science User Facility supported under Contract DE-AC02-06CH11357, provided by the Innovative and Novel Computational Impact on Theory and Experimen (INCITE) program. This work was supported by the Midwest Integrated Center for Computational Materials (MICCoM) as part of the Computational Materials Science Program funded by the US Department of Energy, Office of Science, Basic Energy Sciences, and Materials Sciences and Engineering Division, through Argonne National Laboratory, under Contract No. DE-AC02-06CH11357. A.Y. gratefully acknowledges support from the National Institute of Allergy and Infectious Diseases of the NIH under grant F32 AI150208.

\section{Author contributions}

E.M.Y.L., J.J.d.P. and G.G. designed research. E.M.Y.L. performed research. E.M.Y.L. and A.Y. contributed methods, simulation code, and analytic tools. E.M.Y.L. and A.Y. analyzed data. E.M.Y.L., A.Y., J.J.d.P. and G.G. wrote the paper.

\section{Competing interests}

The authors declare no competing interests.

\section{Additional information}

Supplementary information The online version contains supplementary material available at https://doi.org/10.1038/s41467-021-26419-0.

Correspondence and requests for materials should be addressed to Juan J. de Pablo or Giulia Galli.

Peer review information Nature Communications thanks Antonino La Magna and the other, anonymous, reviewer(s) for their contribution to the peer review of this work. Peer reviewer reports are available.

Reprints and permission information is available at http://www.nature.com/reprints

Publisher's note Springer Nature remains neutral with regard to jurisdictional claims in published maps and institutional affiliations.

Open Access This article is licensed under a Creative Commons Attribution 4.0 International License, which permits use, sharing, adaptation, distribution and reproduction in any medium or format, as long as you give appropriate credit to the original author(s) and the source, provide a link to the Creative Commons license, and indicate if changes were made. The images or other third party material in this article are included in the article's Creative Commons license, unless indicated otherwise in a credit line to the material. If material is not included in the article's Creative Commons license and your intended use is not permitted by statutory regulation or exceeds the permitted use, you will need to obtain permission directly from the copyright holder. To view a copy of this license, visit http://creativecommons.org/ licenses/by/4.0/.

(C) The Author(s) 2021 